\title{
The infalling photon, the infalling particle, and the observer at rest near the horizon of a black hole
}

\author{
Francisco D. Mazzitelli \\ Centro Atómico Bariloche and Instituto Balseiro, \\ Comisión Nacional de Energía Atómica, 8400 Bariloche, Argentina.
}

\begin{abstract}
When a massive test particle or a photon fall radially into a black hole, their energy, as measured by a static observer located very close to the horizon, diverges. In introductory courses on General Relativity, this fact gives rise to questions about the reality of this divergence, and its eventual effect on the geometry of the black hole. We address these concerns and show that, eventually, it is the observer at rest who may induce corrections to the metric, unless its mass is crucially small when located near the horizon.
\end{abstract}




\section{INTRODUCTION}

The Schwarzschild geometry is a static spherically symmetric solution of the vacuum Einstein's equations. In standard (or Schwarzschild) coordinates it is given by [1]

$$
d s^{2}=-c^{2}\left(1-\frac{2 M G}{c^{2} r}\right) d t^{2}+\frac{d r^{2}}{\left(1-\frac{2 M G}{c^{2} r}\right)}+r^{2}\left(d \theta^{2}+\sin ^{2} \theta d \phi^{2}\right)
$$

(from now on we will set the velocity of light $c=1$ ). Despite its simple appearance, there are no elementary derivations based on Newtonian gravity and special relativity [2]; to derive it, one has to consider the full Einstein's equations. The degree of difficulty in solving these equations depends crucially on the coordinates chosen [3].

As is evident the metric has a singularity at the Schwarzschild radius $R_{S}=2 G M$, that defines the event horizon, but this turns out to be a problem associated to the coordinates. Schwarzschild coordinates are valid and describe a spherically symmetric black hole as long as one considers $r>R_{S}$. The singularity of the coordinates at $r=R_{S}$ has some consequences, as a huge redshift of photons emitted by a source that is near the horizon and observed far from the black hole, a hint of the fact that the proper time for observers at infinity, $t$, is not an adequate coordinate to describe the geometry near the horizon. Traditional alternatives discussed in textbooks are Eddington-Finkelstein (EF) and Kruskal-Szekeres (KS) coordinates. Other interesting option is to use Painlevé -Gullstrand coordinates [4].

Let us consider an observer at rest (OAR) at a given radius $r=R$ (coordinates $\theta$ and $\phi$ are also fixed). Assume that a photon is emitted far from the horizon with a frequency $\omega_{\infty}$. The OAR will determine its frequency as $\omega_{I P}^{(O A R)}=\omega_{\infty} / \sqrt{1-R_{S} / R}$, and suffers a very large blueshift (the subindex IP stands for infalling photon). The frequency measured by the static observer diverges as $R \rightarrow R_{S}$. In the same way, the velocity of an infalling particle that is initially at rest far from the horizon, measured by the OAR, is given by $v_{R}=\sqrt{R_{S} / R}$ and tends to the speed of light as $R \rightarrow R_{S}$. Its energy diverges in this limit (these results are reviewed in the next section).

In introductory courses on General Relativity the students wonder whether this huge energy is real: Could this energy kill the rest observer? Can this energy be used? Why this energy does not modify Schwarzschild geometry? Or does it? The first two questions have immediate trivial answers: it is the energy measured by a rest observer, and therefore it is real and can be used as any other type of energy by the OAR. The last two questions could 
be rephrased in terms of the eventual backreaction of the test particle over the metric. As we will see, their answers pinpoints the advantages of using different sets of coordinates, and shed light on some conceptual issues related to the measurements performed by different observers near the horizon.

There is an analogous situation in flat spacetime that illustrates the potential problem: in a given coordinate system, assume there is a test point-particle [5] of mass $m$ which is at rest. For sufficiently small mass, spacetime is almost flat. Outside the particle, the metric is given by the Schwarzschild metric with mass $m$. Consider now a second particle with the same mass but moving with velocity $v$ with respect to the first one. Its energy-momentum tensor will be given by

$$
T^{\mu \nu}(t, \vec{x})=\frac{m}{u^{0}} \delta^{(3)}\left(\vec{x}-\vec{x}_{P}(t)\right) u^{\mu} u^{\nu}=\frac{m}{\sqrt{1-v^{2}}} \delta^{(3)}\left(\vec{x}-\vec{x}_{P}(t)\right) \frac{d x^{\mu}}{d t} \frac{d x^{\nu}}{d t},
$$

where $\vec{x}_{P}(t)$ denotes the trajectory of the moving particle and $u^{\mu}=d x^{\mu} / d \tau$ the components of its four-velocity (the explicit form of the energy-momentum tensor for a point-particle can be derived from its action, see Eqs.(20)-(22) below). Note that, for any fixed value of $m$, no matter how small, the components of the energy-momentum tensor become bigger and bigger as $v \rightarrow 1$, and the ultrarelativistic particle could modify the spacetime metric (i.e. it could produce a strong gravitational field as measured by an observer comoving with the first particle). For instance, in the limit $m \rightarrow 0$ and $v \rightarrow 1$, with $m v / \sqrt{1-v^{2}} \rightarrow p$, the spacetime is described by a shockwave with nonvanishing curvature on the plane that moves with the particle and is perpendicular to its velocity (this is the well-known Aichelburg-Sexl geometry [6]). In this system of coordinates, the curvature produced by the particle at rest is of order $m G$, while the one produced by the moving particle is of order $p G$, that could be much larger (there are interesting geometrical effects produced by the shockwave [7]). Will the infalling particle or the infalling photon produce a similar modifications to the geometry of the black hole?

The article is organized as follows. In Section II we review the computation of the frequency of the IP and of the energy of the free falling particle as measured by the OAR. In Section III we consider the point of view of a free falling observer (FFO). We compute the frequency of the IP in this reference frame. We first consider the case in which the IP and the FFO meet at $r>R_{S}$, and then generalize the results to arbitrary values of $r$. In the latter case, the use of coordinates that are regular at the horizon becomes crucial, and the 
calculation is done in EF coordinates. In Section IV we discuss the eventual backreaction on the geometry produced by either the FFO, the IP, or the OAR. To do this, we compute the components of the energy-momentum tensor for each of them, near the horizon. Once more, the use of regular coordinates is compelling, and the calculation is performed in KS coordinates. We show that, in the limit $r \rightarrow R_{S}$, the components of the energy-momentum tensor remain bounded for both the FFO and the IP (the latter case is discussed in the Appendix). On the contrary, for the OAR, the energy-momentum tensor is divergent in this limit. Section V contains the conclusions of our work: neither the FFO nor the IP will induce modifications to the black hole geometry, as long as the rest mass of the FFO and the initial energy of the IP are much smaller than the black hole's mass. However, the OAR will produce a backreaction, unless its mass tends to zero as $R \rightarrow R_{S}$.

\section{THE OBSERVER AT REST'S POINT OF VIEW}

As a warm up, we remind the reader the well known OAR's point of view, discussed in many textbooks. Since the OAR has a fixed coordinate $r=R$, its four-velocity is given by

$$
\mathbf{u}_{O A R}=\left(\frac{d t}{d \tau}, 0,0,0\right)=\frac{1}{\sqrt{1-\frac{R_{S}}{R}}}(1,0,0,0)
$$

The energy of an object with four-momentum $\mathbf{p}$ as measured by the OAR is given by $E^{(O A R)}=-\mathbf{p} \cdot \mathbf{u}_{O A R}$.

Let us first consider the IP. As it is a massless particle, its four-momentum $\mathbf{p}_{I P}$ satisfies $\mathbf{p}_{I P} \cdot \mathbf{p}_{I P}=0$. Moreover, the metric is static, which implies that $g_{0 \alpha} p_{I P}^{\alpha}=g_{00} p_{I P}^{0}$ is a constant, that we identify with (minus) the energy at infinity, that is, $\hbar \omega_{\infty}$. These two conditions imply

$$
\mathbf{p}_{I P}=\hbar \omega_{\infty}\left(\frac{1}{1-\frac{R_{S}}{R}},-1,0,0\right)
$$

and therefore, when $r=R$ :

$$
\omega_{I P}^{(O A R)}=\frac{E_{I P}^{(O A R)}}{\hbar}=-\frac{1}{\hbar} \mathbf{p}_{I P} \cdot \mathbf{u}_{O A R}=\frac{\omega_{\infty}}{\sqrt{1-\frac{R_{S}}{R}}}
$$

which is the standard result for the gravitational redshift (blueshift in this case).

Let us now consider the infalling particle of rest mass $m$, that from now on will be named 
the FFO. If the FFO starts falling at rest from $r \rightarrow \infty$, its four-momentum reads

$$
\mathbf{p}_{F F O}=m \mathbf{u}_{F F 0}=m\left(\frac{1}{1-\frac{R_{S}}{r}},-\sqrt{\frac{R_{S}}{r}}, 0,0\right) .
$$

This can be derived from $\mathbf{p}_{F F O} \cdot \mathbf{p}_{F F O}=-m^{2}$ and the conservation of energy $g_{00} p_{F F O}^{0}=-m$. As a consequence, the energy of the FFO measured by the OAR is given by

$$
E_{F F O}^{(O A R)}=-\mathbf{p}_{F F O} \cdot \mathbf{u}_{O A R}=\frac{m}{\sqrt{1-\frac{R_{S}}{R}}},
$$

that corresponds to a relativistic particle with velocity $v_{R}=\sqrt{R_{S} / R}$. The velocity of the FFO measured by the OAR tends to 1 , and the energy diverges, as $R \rightarrow R_{S}$.

\section{THE FREE-FALLING OBSERVER'S VIEW}

We now consider the point of view of the FFO. We will first compute the energy of the IP as measured by the FFO assuming that they meet at $r>R_{s}$, and that the FFO is initially at rest at infinity. Then, we will generalize the calculation for arbitrary values of $r$ and of the initial energy of the FFO.

If the FFO and the IP meet at $r>R_{S}$, a simple way to compute the energy of the IP measured by the FFO is to realize that the measurements of the FFO and of the OAR at $r=R$ can be connected by a Lorentz transformation [8], a boost with velocity $v_{R}$. Therefore, the energy of the IP measured by the FFO, $\omega_{I P}^{(I O)}$, is related to the energy measured by the OAR by a Doppler shift factor $\sqrt{\left(1-v_{R}\right) /\left(1+v_{R}\right)}$

$$
\omega_{I P}^{(F F O)}=\omega_{I P}^{(O A R)} \sqrt{\frac{1-v_{R}}{1+v_{R}}}=\frac{\omega_{\infty}}{1+\sqrt{\frac{R_{S}}{R}}},
$$

where we used Eq.(5) and $v_{R}=\sqrt{R_{S} / R}$. It is a simple and instructive exercise to obtain the same result by projecting the four-momentum of the IP on the four-velocity of the FFO, i.e. $\omega_{I P}^{(F F O)}=-\frac{1}{\hbar} \mathbf{p}_{I P} \cdot \mathbf{u}_{F F O}$.

Note that the energy of the photon is finite when measured by a FFO even if they meet at the horizon. This is a consequence of the cancellation between the gravitational blueshift and the Doppler redshift associated with a relative velocity which is close to the speed of light [9]. Using similar arguments, one can compute the energy and velocity of the OAR as measured by the FFO. Of course, there is no need for additional calculations, because 
$v_{R}$ is the relative velocity between them, that is univocally defined by the scalar product $\mathbf{u}_{F F O} \cdot \mathbf{u}_{O A R}[12]$. The energy of the OAR, measured by the FFO, is indeed also infinite as $R \rightarrow R_{S}$.

We will now generalize the above results for arbitrary valu es of the meeting point $r$. If the FFO and the IP meet beyond the horizon, in order to compute $\omega_{I P}^{(F F O)}$ it is necessary to use a different set of coordinates, to avoid the singularity of the Schwarzschild coordinates at the horizon. We will use the EF coordinates, which trade the coordinate $t$ by a new coordinate $v$ defined as [13]

$$
v=t+r+R_{S} \log \left|\frac{r}{R_{S}}-1\right|
$$

In the new coordinates, the spacetime interval reads

$$
d s^{2}=-\left(1-\frac{R_{S}}{r}\right) d v^{2}+2 d v d r+r^{2}\left(d \theta^{2}+\sin ^{2} \theta d \phi^{2}\right) .
$$

Note that the metric is regular at the horizon.

Light cones associated to radially infalling photons are defined by $d v=0$. Therefore $p_{I P}^{v}=0$ and the only non-vanishing contravariant component of the four-momentum is $p_{I P}^{r}$. As the metric does not depend on the coordinate $v, g_{v \alpha} p_{I P}^{\alpha}=p_{I P}^{r}$ turns out to be constant, i.e. (minus) the energy of the IP at infinity, $\hbar \omega_{\infty}$.

The four-velocity of the FFO reads

$$
\mathbf{u}_{F F O}=\left(\frac{d v}{d \tau}, \frac{d r}{d \tau}, 0,0\right)
$$

and the two non-vanishing components can be obtained from the conservation law

$$
-e_{\infty}=g_{v \alpha} u_{F F O}^{\alpha}=-\left(1-\frac{R_{S}}{r}\right) \frac{d v}{d \tau}+\frac{d r}{d \tau}
$$

and the normalization

$$
\mathbf{u}_{F F O} \cdot \mathbf{u}_{F F O}=-1=-\left(1-\frac{R_{S}}{r}\right)\left(\frac{d v}{d \tau}\right)^{2}+2 \frac{d v}{d \tau} \frac{d r}{d \tau} .
$$

The constant $e_{\infty} \geq 1$ is the energy per unit mass of the FFO, and equals 1 when it is initially at rest. For the sake of completeness, we will compute $\omega_{I P}^{(F F O)}$ for an arbitrary initial energy.

The frequency of the IP, measured by the FFO, when they meet at $r$, is given by

$$
\omega_{I P}^{(F F O)}=-\frac{1}{\hbar} \mathbf{u}_{F F O} \cdot \mathbf{p}_{I P}=\omega_{\infty} \frac{d v}{d \tau} .
$$


We can compute $d v / d \tau$ by combining Eqs.(12) and (13). The combination gives the following quadratic equation for $d v / d \tau$

$$
\left(1-\frac{R_{S}}{r}\right)\left(\frac{d v}{d \tau}\right)^{2}-2 e_{\infty} \frac{d v}{d \tau}+1=0
$$

that must be solved with the constraint $d r / d \tau<0$. The result is

$$
\frac{d v}{d \tau}=\frac{\omega_{I P}^{(F F O)}}{\omega_{\infty}}=\frac{1}{1-\frac{R_{S}}{r}}\left[e_{\infty}-\sqrt{e_{\infty}^{2}-1+\frac{R_{S}}{r}}\right]
$$

where $r$ is the radial coordinate at the meeting point. This expression is valid for all positive values of $r$ and tends to $1 /\left(2 e_{\infty}\right)$ as $r \rightarrow R_{S}$. When the FFO is initially at rest $\left(e_{\infty}=1\right)$ we

recover the previous result Eq.(8) setting $r=R$. It is interesting to remark that $\omega_{I P}^{(F F O)} \rightarrow 0$ when the FFO and the IP meet near the black hole's singularity at $r \rightarrow 0$.

The fact that the FFO measures a finite value for the frequency of the IP at (and beyond) the horizon, suggests that the IP should not introduce any backreaction on the geometry.

\section{THE ENERGY-MOMENTUM TENSOR IN REGULAR COORDINATES}

From the FFO point of view, it is the OAR who has almost infinite energy. And viceversa. Then the question is: which of them, if anyone, would produce a backreaction on the geometry? Since we are interested in what happens near the horizon, it is again mandatory to use coordinates that are regular there.

To evaluate the effect on the metric, one could solve Einstein's equations using the energymomentum tensor with either the FFO, or the IP, or the OAR, as a source, eventually assuming that the metric describes a small perturbation of the Schwarzschild geometry in those coordinates. Instead of doing this, we will just evaluate the order of magnitude of the components of the energy-momentum tensor. We are admittedly using this very crude and simplistic approach, estimating the backreaction through the magnitude of the source of Einstein's equations and neglecting radiation-reaction forces [14]. This will be enough for our purposes, which is to provide an elementary discussion in the context of introductory courses on General Relativity. 
We will use KS coordinates $(T, X)$, that for $r>R_{S}$ are related with $(t, r)$ by [15]

$$
\begin{aligned}
T & =\sqrt{\frac{r}{R_{S}}-1} e^{\frac{r}{2 R_{S}}} \sinh \left[\frac{t}{2 R_{S}}\right] \\
X & =\sqrt{\frac{r}{R_{S}}-1} e^{\frac{r}{2 R_{S}}} \cosh \left[\frac{t}{2 R_{S}}\right] .
\end{aligned}
$$

The angular coordinates $(\theta, \phi)$ remain unchanged. In these coordinates, the spacetime interval reads

$$
d s^{2}=\frac{4 R_{S}^{3}}{r} e^{-\frac{r}{R_{S}}}\left(-d T^{2}+d X^{2}\right)+r^{2}\left(d \theta^{2}+\sin ^{2} \theta d \phi^{2}\right),
$$

where $r$ should be considered as a function $r(T, X)$ defined implicitly by Eqs.(17). Indeed we have

$$
\left(\frac{r}{R_{S}}-1\right) e^{\frac{r}{R_{S}}}=X^{2}-T^{2}
$$

which shows that in $\mathrm{KS}$ coordinates the horizon is located at $X=T$ (this corresponds to $R \rightarrow R_{S}$ and $t \rightarrow \infty$ in Schwarzschild coordinates). Note that,when $d \theta=d \phi=0$, the light cones are defined by $d T / d X= \pm 1$.

Assume that the mass $m$ of the FFO and of the OAR is small enough to be considered a test particle when it is at rest far from the horizon. We will compute the components of the energy-momentum tensor associated to the mass, using KS coordinates, for both cases: when the mass $m$ falls from very far (FFO) and when the mass is located at $R \simeq R_{S}(\mathrm{OAR})$. As already mentioned, these components should be used as a source in Einstein's equations to evaluate the backreaction of the particle on the metric. If their values are always $O(m)$, we expect no appreciable backreaction on the metric. If some of the components blow up near the horizon, then the particle could modify the background geometry [16].

The components of the energy-momentum tensor can be derived by taking the derivative with respect to the metric of the action for the point-particle:

$$
S_{P}=-m \int d \tau \sqrt{-g_{\alpha \beta} u^{\alpha} u^{\beta}}
$$

and is given by [17]

$$
T^{\mu \nu}(x)=\frac{2}{\sqrt{g}} \frac{\delta S_{P}}{\delta g_{\mu \nu}(x)}=m \int \frac{d \tau}{\sqrt{g(x)}} \delta^{(4)}\left(x-x_{P}(\tau)\right) u^{\mu} u^{\nu},
$$

where $u^{\mu}$ is the four-velocity of the particle and $x_{P}(\tau)$ its world line. Performing the integral over proper time we obtain

$$
T^{\mu \nu}(T, \vec{x})=\frac{m}{\sqrt{g}} \delta^{(3)}\left(\vec{x}-\vec{x}_{P}(T)\right) \frac{u^{\mu} u^{\nu}}{u^{0}} .
$$


As we already know the four-velocities of the observers in Schwarzschild coordinates, it is enough to consider the transformation rule for four-vectors under a change of coordinates

$$
u^{\alpha}=\frac{\partial x^{\alpha}}{\partial x^{\prime} \beta} u^{\prime \beta}
$$

Here primed (non-primed) coordinates refer to Schwarzschild (KS) coordinates. For both the OAR and the FFO we will have

$$
\begin{aligned}
& u^{T}=\frac{\partial T}{\partial t} \frac{d t}{d \tau}+\frac{\partial T}{\partial r} \frac{d r}{d \tau} \\
& u^{X}=\frac{\partial X}{\partial t} \frac{d t}{d \tau}+\frac{\partial X}{\partial r} \frac{d r}{d \tau}
\end{aligned}
$$

For the OAR and by Eq.(3) we have $d r / d \tau=0$ and $d t / d \tau=1 / \sqrt{1-R_{S} / R}$. Using the relation between KS and Schwarzschild coordinates, given in Eqs.(17), it is straightforward to obtain

$$
\begin{aligned}
& u_{O A R}^{T}=\frac{X}{2 R_{S}} \frac{1}{\sqrt{1-\frac{R_{S}}{R}}} \\
& u_{O A R}^{X}=\frac{T}{2 R_{S}} \frac{1}{\sqrt{1-\frac{R_{S}}{R}}},
\end{aligned}
$$

where $R=R(X, T)$ is defined implicitly by Eq.(19).

On the other hand, when the FFO meets the OAR at $r=R$, by Eq.(6) we have $d r / d \tau=$ $-\sqrt{R_{s} / R}$ and $d t / d \tau=1 /\left(1-R_{S} / R\right)$. Therefore,

$$
\begin{aligned}
& u_{F F O}^{T}=\frac{1}{2 R_{S}\left(1-\frac{R_{S}}{R}\right)}\left(X-T \sqrt{\frac{R_{S}}{R}}\right) \\
& u_{F F O}^{X}=\frac{1}{2 R_{S}\left(1-\frac{R_{S}}{R}\right)}\left(T-X \sqrt{\frac{R_{S}}{R}}\right) .
\end{aligned}
$$

Eqs.(25) show that the components of the four-velocity of the OAR grow as the observer is located near the horizon $\left(X \simeq T, R \simeq R_{S}\right)$. Being proportional to $1 / \sqrt{1-R_{S} / R}$, the components of the energy-momentum tensor grow accordingly. To avoid this, the mass of the OAR should be chosen smaller and smaller as $R \rightarrow R_{S}$. Note that in this case, the trajectory of the OAR is almost on the surface of the light cone:

$$
\left.\frac{d X}{d T}\right|_{O A R}=\frac{u_{O A R}^{X}}{u_{O A R}^{T}}=\frac{T}{X} \simeq 1 .
$$

On the other hand, from Eqs.(26) one can show that the components of the four-velocity of the FFO, and therefore also those of its energy-momentum tensor, remain finite as she/he 
crosses the horizon. To see this it is necessary to evaluate in detail the limit $R \rightarrow R_{S}$. Assume that $R / R_{S}=1+\delta$ with $\delta \ll 1$. Taking into account that $X \rightarrow T$ as $\delta \rightarrow 0$, from Eq.(19) we have

$$
X-T \simeq \frac{e \delta}{2 X}+O\left(\delta^{2}\right)
$$

Inserting this into Eqs.(26) we obtain, to zeroth order in $\delta$,

$$
\begin{aligned}
& u_{F F O}^{T} \simeq \frac{X}{4 R_{S}}\left(1+\frac{e}{X^{2}}\right) \\
& u_{F F O}^{X} \simeq \frac{X}{4 R_{S}}\left(1-\frac{e}{X^{2}}\right),
\end{aligned}
$$

and therefore

$$
\left.\frac{d X}{d T}\right|_{F F O}=\frac{u_{F F O}^{X}}{u_{F F O}^{T}} \simeq \frac{1-\frac{e}{X^{2}}}{1+\frac{e}{X^{2}}},
$$

whose absolute value is less than one for any finite value of the coordinate $X \simeq T$. These equations show that, unlike the OAR, when the FFO reaches the horizon the components of its four-velocity remain bounded [18]. Moreover, its trajectory is inside the local light cone even when it is on the horizon (as it should).

It is instructive to check that the components of the energy-momentum tensor of the IP remain $O\left(\hbar \omega_{\infty}\right)$ as the photon crosses the horizon. The details are presented in the Appendix.

The results of this Section show that neither the FFO nor the IP will induce modifications to the metric of the black hole. The mass of the OAR, instead, should tend to zero as $R \rightarrow R_{S}$, in order to avoid the growth of the components of its energy-momentum tensor.

\section{DISCUSSION}

For a test particle, the energy-momentum tensor is proportional to its mass and, as long as its components are $O(m)$ along the trajectory and $m$ is sufficiently small, it will not produce sizeable modifications to the metric. Computing the energy-momentum tensor for the FFO in regular coordinates, we have seen that its components remain of order $m$ all along the trajectory. A similar argument applies for low-energy massless particles (see the Appendix below). Therefore, the infalling test particle and the infalling low-energy photon

will not produce corrections to the Schwarzschild geometry. On the other hand, for the OAR the components of the energy-momentum tensor diverge as $R \rightarrow R_{S}$, unless its mass 
is chosen to be smaller and smaller in this limit. In other words, if a small-mass spacecraft falls freely into a black hole, it will not modify significantly the metric. However, if at some point the engine is turned on and it remains at a fixed $R / R_{S}=1+\delta$ with $\delta$ sufficiently small, it will. Even neglecting the gravitational waves produced until arriving at $r=R$. An alternative observation that reinforces these results is the fact that the proper acceleration of the OAR, given by [19]

$$
a_{O A R}=\sqrt{g_{\alpha \beta} \frac{d u_{O A R}^{\alpha}}{d \tau} \frac{d u_{O A R}^{\beta}}{d \tau}}=\frac{R_{S}}{R^{2}} \frac{1}{\sqrt{1-\frac{R_{s}}{R}}},
$$

also diverges as $\delta \rightarrow 0$.

The results we obtained may not be intuitive since, at first sight, one could assume that the FFO becomes ultrarelativistic when approaching the horizon. Indeed, there seems to be some confusion in the literature regarding the value of the velocity of the FFO when reaching the horizon. While it is true that the OAR will determine that the velocity of the FFO is close to the velocity of light, this is not true for all observers [20], as illustrated by Eq.(301). The strength of the gravitational field is also observer-dependent [21].

An analysis based on coordinates that are regular at the horizon shows that the ultrarelativistic assumption for the FFO is wrong. In fact, in these coordinates the path of the FFO lies well inside the light cone, while the path of the OAR is close to the light cone surface (the horizon!).

\section{ACKNOWLEDGEMENTS}

This research was supported by ANPCyT, CONICET, and UNCuyo. I would like to thank the International Centre for Theoretical Physics, Trieste, Italia, for hospitality during the initial stages of this work.

\section{APPENDIX : THE ENERGY-MOMENTUM TENSOR FOR THE INFALLING PHOTON}

In this Appendix we show that the components of the energy-momentum tensor for the IP remain finite as the photon reaches the horizon of the black hole. To begin with, we 
note that the energy momentum tensor for a massive particle in Eq.(22) can be rewritten in terms of its four-momentum as

$$
T^{\mu \nu}(T, \vec{x})=\frac{1}{\sqrt{g}} \delta^{(3)}\left(\vec{x}-\vec{x}_{P}(T)\right) \frac{p^{\mu} p^{\nu}}{p^{0}} .
$$

This expression is also valid for massless particles, as can be guessed from the fact that one can obtain the four-momentum of a massless particle as the limit $m \rightarrow 0, v \rightarrow 1$ of the four-momentum of a massive particle, assuming that $m / \sqrt{1-v^{2}}$ remains finite in this limit,

$$
\begin{aligned}
& p_{m \neq 0}^{0}=\frac{m}{\sqrt{1-v^{2}}} \rightarrow p_{m=0}^{0} \\
& \vec{p}_{m \neq 0}=\frac{m \vec{v}}{\sqrt{1-v^{2}}} \rightarrow \vec{p}_{m=0} .
\end{aligned}
$$

Therefore, when the IP is located at $\vec{x}_{I P}(T)$ we have

$$
T_{I P}^{\mu \nu}(T, \vec{x})=\frac{1}{\sqrt{g}} \delta^{(3)}\left(\vec{x}-\vec{x}_{I P}(T)\right) \frac{p_{I P}^{\mu} p_{I P}^{\nu}}{p_{I P}^{0}} .
$$

We will compute the components of the four-momentum of the IP near the horizon, using KS coordinates. As for the OAR and the FFO, we use the transformation rule for four-vectors under a coordinate transformation (see Eqs.(24)). In this case we have

$$
\begin{aligned}
& p_{I P}^{T}=\frac{\partial T}{\partial t} p_{I P}^{t}+\frac{\partial T}{\partial r} p_{I P}^{r} \\
& p_{I P}^{X}=\frac{\partial X}{\partial t} p_{I P}^{t}+\frac{\partial X}{\partial r} p_{I P}^{r} .
\end{aligned}
$$

The components of the four-momentum in Schwarzschild coordinates are given in Eq.(4). Inserting this equation into Eqs.(35), and using the relations between KS and Schwarzschild coordinates, given in Eqs.(17), we obtain

$$
p_{I P}^{T}=-p_{I P}^{X}=\frac{\hbar \omega_{\infty}}{2 R_{S}\left(1-\frac{R_{S}}{R}\right)}(X-T)
$$

From Eq.(28) we see that $X-T=O\left(1-R_{S} / R\right)$, and therefore the components of the fourmomentum of the IP remain finite and of order $\hbar \omega_{\infty}$ as $R \rightarrow R_{S}$. The same happens with the components of its energy-momentum tensor.

[1] J.B. Hartle, Gravity: An introduction to Einstein's General Relativity, Addison Wesley, San F rancisco, (2003), §9.1; B.F. Schutz, A first course in general relativity, Cambridge University Press, Cambridge, (1985), §10.4; S. Carroll, Spacetime and Geometry: An Introduction to General Relativity, Cambridge University Press, Cambridge, (2019), §5.1. 
[2] R.P. Gruber et al, "The impossibility of a simple derivation of the Schwarzschild metric", Am. J. Phys. 56, 265-269 (1988).

[3] P. Fromholz, E. Poisson, and C. M. Will, "The Schwarzschild metric: It's the coordinates, stupid!" Am. J. Phys. 82, 295-300 (2014).

[4] K. Martel and E. Poisson, "Regular coordinate systems for Schwarzschild and other spherical spacetimes", Am. J. Phys. 69, 476-480 (2001).

[5] For simplicity we consider a "point"-particle. By this we mean a particle whose size is much smaller than any other relevant scale in our discussion. To be considered a test particle, its radius $\left(R_{P}\right)$ should be much larger than the Schwarzschild radius associated to the particle, that is $R_{P} \gg 2 m G$. Of course we will also assume $m \ll M$, the mass of the black hole.

[6] P.C. Aichelburg and R.U. Sexl, "On the gravitational field of a massless particle", Gen. Relat. Gravit. 2 (4), 303-312 (1971).

[7] T. Dray and G. t'Hooft, "The gravitational shock wave of a massless particle", Nucl. Phys. B 253, 173-188 (1985).

[8] E.F. Taylor and J.A. Wheeler, Exploring black holes: Introduction to General Relativity, Addison Wesley, San Francisco, (2000), page 2-34.

[9] The factorization of the total frequency change into gravitational and kinematical (Doppler) terms has been discussed at length in the literature [10]. Formulas that generalize Eq.(8) for the case in which the two observers are in motion have also been derived [11], and are relevant to analyze the communication between them in the vicinity of the horizon. Eq.(8) will be enough for our discussion.

[10] A. Radosz, A.T. Augousti, and K. Ostasiewicz, "The Doppler shift in a Schwarzschild spacetime", Phys. Lett. A373, 801-803 (2009).

[11] A. T. Augousti, M. Gawelczyk, A. Siwek, and A. Radosz, "Touching ghosts: observing free fall from an infalling frame of reference into a Schwarzschild black hole", Eur. J. Phys 33, 1-11 (2012); A.V. Toporensky, and O.B. Zaslavskii, "Redshift of a photon emitted along the black hole horizon", Eur. Phys. J. C 77, 179 (2017).

[12] The relative velocity is univocally defined for observers at the same spacetime point. For generalizations see V.J. Bolós, Commun. Math. Phys. 273, 217-236 (2007).

[13] See J.B. Hartle, Ref. [1], §12.1.

[14] F. Rohrlich, "The self-force and radiation reaction", Am. J. Phys. 68, 1109-1112 (2000). 
[15] See J.B. Hartle, Ref. [1], §12.3.

[16] A technical remark: although both KS and EF coordinates are regular at the horizon, for this discussion we prefer the use of KS coordinates, because all components of the metric are non-vanishing at the horizon. In this way, it is possible to avoid concerns regarding which components $T^{\mu \nu}, T_{\nu}^{\mu}$ or $T_{\mu \nu}$ should be computed.

[17] S. Weinberg, Gravitation and Cosmology, J. Wiley, New York (1972), §12.2 ; M. Katanaev, "Point massive particle in General Relativity", Gen. Rel. Grav. 45, 1861-1875 (2013)

[18] This has already been pointed out in the Appendix of A.T. Augousti et al, "On the speed of a test particle inside the Schwarzschild event horizon and other kinds of black holes", General Relativity and Gravitation 50, 131 (2018).

[19] N.A. Doughty, "Acceleration of a static observer near the event horizon of a static isolated black hole", Am. J. Phys. 49, 412-416 (1981).

[20] See P. Crawford, and I. Tereno, "Generalized observers and velocity measurements in General Relativity", General Relativity and Gravitation 34, 2075-2088 (2002), and references therein. See also Augousti et al [18]

[21] B. Mashhoon, "On the strength of a gravitational field", Phys. Lett. A 163, 7-14 (1992). 